\title{
A Hierarchical P2PSIP Architecture to support Skype-like services
}

\author{
Isaias Martinez-Yelmo, Carmen Guerrero, Ruben Cuevas \\ Departamento de Ingeniería Telemática \\ Universidad Carlos III de Madrid \\ Av. Universidad 30. 28911 Leganés. Madrid (Spain) \\ \{imyelmo, guerrero, rcuevas\}@it.uc3m.es
}

\author{
Andreas Mauthe \\ Computing Department, InfoLab 21 \\ Lancaster University \\ Lancaster LA1 4WA (UK) \\ andreas@comp.lancs.ac.uk
}

\begin{abstract}
A Hierarchical DHT Overlay Architecture based on P2PSIP is proposed to support a Skype-like service. The IETF P2PSIP Working Group is standardising a protocol to support any DHT in order to deploy services inside a domain. We extend its functionality to allow the interaction between peers of different domains. Furthermore, we perform an analysis of the Routing Performance and Resource Consumption under a Skype-like scenario where VoIP calls are more likely to happen among users of the same domain.
\end{abstract}

\section{Introduction}

Nowadays, one of the most widely researched topics is the provision of Multimedia services on the Internet. However, due to their requirements, currently there is a lack of development effort. Although, some services are successful, (Skype is a good example), they are not easy to design and develop. Thus, additional research and tools are needed in order to deploy easily Multimedia added value services.

In this paper, we examine the benefits of adopting a Hierarchical Overlay Network to develop a Skype-like service. Skype is a widely used application, but it is a proprietary solution and a standardised solution is preferred. Some details about Skype can be found in [6] or [16]. In order to obtain a low cost Skype-like solution, the IETF P2PSIP Working Group has been created to work on a similar but standardised alternative. The idea is to provide a decentralised architecture that replaces the functionalities of proxy and registrar SIP servers with a peer-to-peer overlay network [2]. The result of this work is the P2PSIP protocol [7] that is being defined to support any kind of DHT overlay network such as Content Addressable Network (CAN) [14] or Kademlia [11] where the desired information can



Figure 1. Hierarchical Overlay Architecture

be stored in a decentralised fashion. Furthermore, the connections would be NAT Traversal capable using the ICE protocol [15] based on STUN and TURN relays published as services in the P2P network. However, the connectivity is only possible between peers of the same P2PSIP domain.However, our proposal enables the connectivity between different domains.

This proposal is shown in Fig.1 where different P2PSIP domains deploy their own overlay and global connectivity between the different domains is obtained with an Interconnection Overlay in which each domain is represented by at least one super-peer. This approach has a number of advantages: first of all, VoIP calls usually are more frequent among peers that belong to the same domain. Several examples include intradomain VoIP calls inside the headquarters of a private company or VoIP calls and Instant Messaging between people subscribed to some Community/Social Network. Second, a hierarchical architecture allows fault isolation since if a network failure happens in the gateway router of a company supporting P2PSIP for its VoIP calls, it does not affect other P2PSIP domains. The Interconnection Overlay is only slightly affected since only this super-peer is not reachable. In addition, 
the advantage of a better scalability is obtained, which is a known fact in hierarchical architectures [18]. Other well known advantages are explained in literature [1].

This paper addresses how a Hierarchical DHT Overlay Network can be used to interconnect different P2PSIP domains and it studies analytically the Routing Performance in terms of hops to reach the destination.

The rest of the paper is structured as follows. Section 2 presents the proposed hierarchical DHT architecture and how to apply the proposed architecture to provide a global P2P VoIP service. The Routing Performance of this architecture considering the intra-domain hit probability is detailed in section 3. The efficiency of this architecture is explored in the case of a Hierarchical CAN Overlay Network in section 4. Finally, in section 5 the related work associated with Hierarchical Overlay Networks is presented and section 6 presents the conclusions.

\section{Hierarchical DHT Overlay Architecture}

The proposed hierarchical architecture is based on creating two different levels: i) the P2PSIP domains and ii) the Interconnection Overlay. If a peer wants to retrieve information from a different domain, it must route its query to the super-peers, which maintain the reachability between the different domains through the Interconnection Overlay. Thus, any domain is reached through the Interconnection Overlay.

\begin{tabular}{|l|l|}
\hline Prefix ID (n-bits) & Suffix ID(m-bits) \\
\hline
\end{tabular}

Figure 2. Hierarchical ID

The IDs that supports this architecture are composed by two fields a Prefix and a Suffix as it is illustrated in Fig.2. Peers inside a domain use the Suffix ID for the domain overlay network. On the other hand, the super-peers route the queries along the Interconnection Overlay using the Prefix ID. Thus, if a peer needs to find a peer or item with a different Prefix ID, the query is sent to its super-peer. This super-peer routes the query to the super-peer that manages the domain in which the destination peer is placed and finally the query is propagated in the target domain.

Peers obtain several benefits with this approach. The maintenance state is reduced in comparison with the flat counterpart, a peer only has to take care about the peers of its own domain. If a flat overlay network is used to support all the peers of the different domains, the routing tables of all the peers would be larger. With the hierarchical approach, peers have to maintain in average an overlay of $N / K$ peers (where $K$ is the number of domains), whereas in the flat overlay the number of peers is $N$. In fact, the reduction of routing entries with this architecture in the peers is approximately $\frac{\log _{B} K}{\log _{B} N} \cdot 100 \%$ if the routing size has a logarithmic dependency with the number of peers (which is quite common). This reduction not only implies less memory and CPU consumption, but the bandwidth needed for the maintenance of the overlay routing entries is also reduced. This is an advantage in comparison with other proposals like [4], [19]. The drawback is the overload in the super-peers [1], especially in the case of bandwidth and CPU consumption. In this paper, we assume that a mechanism exists to select suitable super-peers [12], [13].

\subsection{Interconnection of P2PSIP domains}

One of the main problems in developing a decentralised architecture the mapping between the available information and the peers in the system. If we consider a VoIP environment, the most usual way of referencing a buddy is using URIs (e.g. user@example.com). Associated with each URI, a Resource contains the user location information.

An user URI can be divided in two fields: user ID (user) and domain ID (example.com), which are separated by @. Thus, this separation can be used to map user URIs to a Hierarchical DHT Overlay Network. The Prefix ID or domain ID is created with the hash of the domain ID: Prefix ID = hash (example.com). The Suffix ID or peer ID is generated with the hash of the URI: Suffix ID = hash (userdexample.com).

Once the mapping is performed, a tuple containing the desired contact information and the generated key is stored on the overlay. This operation is equivalent to the registration in the registrar SIP server in a SIP environment. At this point, the user information is available for the rest of the users. The worst case scenario for retrieving this information occurs when the target buddy is located in an other domain. In this case, the Prefix ID is generated from the domain ID and the Suffix ID from the complete URI. Once this actions are performed, a request is sent to the super-peer of the own domain. Then, the super-peer is able to route the query to the super-peer of the target domain using the Prefix ID. Once the super-peer of target domain receives the query, it forwards the query inside the domain using the Suffix ID. If the contact information is available, it is immediately sent back and the establishment of a Multimedia Session starts. 




Figure 3. Hierarchical P2PSIP Signalling

\subsection{Signalling Exchange}

An example of the signalling on the proposed hierarchical scenario is illustrated in Fig.3, considering the actual status of the P2PSIP protocol [7]. We must take into account several things in order to understand the signalling flow. First of all, when the peer in domain.b requests the information of user1@domain.a, the query in the Fetch message is in plain text. This is because a peer in a domain does not have to know what hash function is being used on the interconnection overlay and what hash function would be used in the other P2PSIP domain, which can have any length and also gives the Prefix ID length. Thus, the super-peer in domain.b performs hash(domain.a) in order to obtain the information of the super-peers in domain.a through the interconnection overlay. Inside this information, the hash used on the other domain is included $\left(\operatorname{hash}_{a}\right)$, and therefore a request for the desired item is correctly formed with the correct length as $\operatorname{hash}_{a}$ (user1@domain.a). Thus, the desired interoperability is obtained. Finally, the peers taking care of the desired Resource-ID answers to the super-peer on domain.a, which forwards this information to superpeer in domain.b. Super-peer in domain.b send the desired Resource-ID to the peer in domain.b. Once this flow finishes, a legacy SIP negotiation is initiated for a VoIP call. We must highlight that Fig. 3 represents only a subset of the real flow where the intermediate hops in each overlay are not shown.

\section{Routing Performance in a Hierarchical Overlay Network for a Skype-like service}

In this section we study the Routing Performance of the architecture proposed. We extend the methodology defined in [10] with a more detailed explanation and model as well as a complete explanation of the considered assumptions. The parameters in the analytical model are defined as follows:

- $K$ : number of P2PSIP domains.

- $M_{i}$ : number of peers in the P2PSIP domain $i$.

- $N$ : all peers in the different P2PSIP domains. It is considered that a peer cannot be attached to multiple domains, hence $N=\sum_{i=1}^{K} M_{i}$.

- $\rho_{i j}$ : probability of launching a query from the P2PSIP domain $i$ to the P2PSIP domain $j$.

- $C(x)$ : number of hops needed to find a super-peer on the interconnection overlay depending on the number of super-peers $x$. It depends on the type of overlay used in the interconnection overlay.

- $D_{i}(x)$ : number of hops needed to find a peer in P2PSIP domain $i$ as function of the number of peers $x$ belonging to the P2PSIP domain.

We assume that all the peers in a P2PSIP domain know their super-peer attached in the interconnection overlay. This assumption implies that only one hop is needed to reach the super-peer.

Taking into account the above definitions, we obtain the Routing Performance (R.P.) of this DHT-based hierarchical overlay network. Let us define the cost of finding a peer on each overlay:

- $D_{i}\left(M_{i}\right)$ : cost of finding a peer on its own domain.

- $C(K)$ : cost of finding a super-peer on the interconnection overlay.

If the probability of obtaining an item in a P2PSIP domain from its super-peer is considered negligible (this can be assumed because the number of superpeers is $N / K$ and $N \gg K$ ), the average Routing Performance for a peer in domain $i$ can be written as follows:

$R P_{i}=\rho_{i i} \cdot D_{i}\left(M_{i}\right)+\sum_{j=1, j \neq i}^{K} \rho_{i j} \cdot\left[1+D_{j}\left(M_{j}\right)+C(K)\right]$

The first term in the previous expression is the cost of searching within the P2PSIP domain of a peer, whereas the second term is the cost for searches in other domains.

Thus, the average number of hops is expressed as:

$$
R P=\frac{1}{N} \cdot \sum_{i=1}^{K} M_{i} \cdot R P_{i}
$$

However, some simplifications can be done in Eq.2 if the number of peers is the same in all domains:

$$
R P=\frac{1}{K} \cdot \sum_{i=1}^{K} \cdot R P_{i}
$$




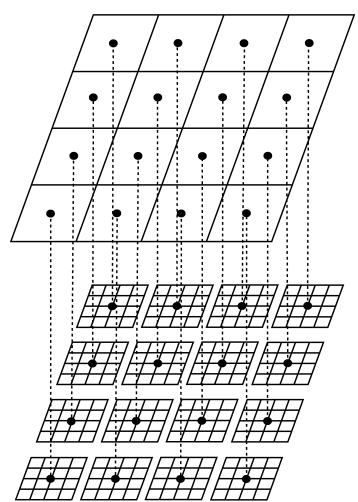

Figure 4. Hierarchical CAN network

Since we considered an equal number of peers in all P2PSIP domains and each look-up in the overlay is randomly independent, the probability of looking for a peer attached to other domain can be equally distributed between all the foreign domains.However, we may still have situations where the probability of looking for a peer in the own P2PSIP domain is different from the probability of looking for a peer in other domains, in which case Eq.1 is expressed as follows:

$R P_{i}=\rho_{i i} \cdot D_{i}(M)+\frac{1-\rho_{i i}}{K-1} \sum_{j=1, j \neq i}^{K}\left[1+D_{j}(M)+C(K)\right]$

This relation is useful for some type of scenarios. For instance, it can be used in a VoIP scenario where the probability of performing a call within the same domain is higher than a call to an external domain.

Finally, if the same overlay is used on all the P2PSIP domains the sum can be eliminated from Eq.4:

$$
R P_{i}=\rho_{i i} \cdot D(M)+\left(1-\rho_{i i}\right) \cdot[1+D(M)+C(K)]
$$

From Eq.3 and Eq.4 we obtain the next equality:

$$
R P=R P_{i}=D(M)+\left(1-\rho_{i i}\right) \cdot[1+C(K)]
$$

We define $\rho_{i i}$ as the intra-domain hit probability and it defines the probability of establishing a connection inside the own domain.

\section{Hierarchical CAN Network}

In this section we study the performance of a hierarchical CAN overlay with two levels, this is shown in Fig.4. According to [14], we have that $C(x)=d_{i} x^{\frac{1}{d_{i}}}$ and $D(x)=d_{l} x^{\frac{1}{d_{l}}}$, so if we use them on Eq.6, we have that the Routing Performance (R.P.) is:

$$
\text { R.P. }=d_{l}(N / K)^{\frac{1}{d_{l}}}+\left(1-\rho_{i i}\right)\left(1+d_{i} K^{\frac{1}{d_{i}}}\right)
$$

Eq.7 represents the Routing Performance for a hierarchical CAN overlay where $d_{l}$ is the number of dimensions for the overlays in each domain and $d_{i}$ is the number of dimensions for the interconnection overlay. Each value can be optimised independently considering the number of peers at each level. Thus, it can be stated that $d_{l}=\ln (N / K)$ and $d_{i}=\ln K$ (these values can be obtained minimising the Routing Performance of the flat CAN overlay network [14]). However, if the development of a hierarchical CAN based application is considered, it would be reasonable to use the same value $d$ in both levels of the hierarchy, since just one version of CAN needs to be implemented and the code can be easily reused. Eq.8 presents the Routing Performance when only one value of $d$ is used in both layers:

$$
R . P .=d(N / K)^{\frac{1}{d}}+\left(1-\rho_{i i}\right)\left(1+d K^{\frac{1}{d}}\right)
$$

The study that can be applied to this scenario is to optimise the R.P. according to the number of dimensions $d$ when the number of P2PSIP domains and the number of peers per domain is given. This study makes sense since P2PSIP domains are independent entities that cannot be merged or splitted, and therefore an optimisation in relation with $\mathrm{K}$ is not possible and only $d$ can be used to optimise the R.P.

Theorem 1. The value of $d$ that minimises the number of hops on a hierarchical CAN overlay network with $N$ peers and $K$ domains is unique.

Proof:

First we prove the existence of a minimum by deriving Eq. 8 with respect to $d$ :

$$
\begin{aligned}
f^{\prime}(d) & =\left(\frac{N}{K}\right)^{\frac{1}{d}}\left(1-\ln \left(\frac{N}{K}\right)^{\frac{1}{d}}\right)+ \\
& +\left(1-\rho_{i i}\right) K^{\frac{1}{d}}\left(1-\ln K^{\frac{1}{d}}\right)
\end{aligned}
$$

Taking into account that $d \in(1, \infty)$, then:

$$
f^{\prime}(d=1)=\frac{N}{K}\left(1-\ln \frac{N}{K}\right)+\left(1-\rho_{i i}\right) K(1-\ln K)
$$

and 


$$
\lim _{d \rightarrow \infty} f^{\prime}(d)=2-\rho_{i i}
$$

Thus, if $\frac{N}{K}, K>e$, then $f^{\prime}(d=1)<0$ and $f^{\prime}(d=\infty)>0$ and considering that $f^{\prime}$ is a continuous function, we can conclude that one or more solutions exist for $f^{\prime}(d)=0$ in our range of work.

Now we prove the unicity of the solution. Therefore, the second derivate with respect to $d$ is calculated:

$$
\begin{aligned}
f^{\prime \prime}(d) & =\frac{1}{d}\left(\frac{N}{K}\right)^{\frac{1}{d}}\left(\ln \left(\frac{N}{K}\right)^{\frac{1}{d}}\right)^{2}+ \\
& +\left(1-\rho_{i i}\right) \frac{K^{\frac{1}{d}}}{d}\left(\ln K^{\frac{1}{d}}\right)^{2}
\end{aligned}
$$

Taking into account again that $f^{\prime \prime}(d)>0, \forall d \in$ $(1, \infty)$, then $f^{\prime}(d)$ is a monotonic increasing function and there is only one solution of $f^{\prime}(d)=0$ and this value is a minimum.

The result of the theorem is interesting because it assures an optimal value for the number of dimensions $d$ in a hierarchical CAN overlay network. Thus, let us explore if some result can be obtained. We apply the following variable substitution:

$$
A=(N / K)^{\frac{1}{d}}, B=K^{\frac{1}{d}}
$$

If the above substitution is applied in Eq.9, we have:

$$
f^{\prime}(d)=A(1-\ln A)+B(1-\ln B)
$$

Furthermore, if $\mathrm{A}$ and $\mathrm{B}$ are transformed to find a relation between them, we can obtain:

$$
\ln B=\frac{\ln K}{\ln (N / K)} \ln A
$$

Suppose $x=\frac{\ln K}{\ln (N / K)}$, we obtain that that $B=$ $A^{x}$. With this result, we can rewrite Eq.14 as:

$$
f^{\prime}(d)=A(1-\ln A)+\left(1-\rho_{i i}\right) A^{x}\left(1-\ln A^{x}\right)
$$

The bisection method can be used to solve $f^{\prime}(d)=$ 0 . The solution gives the optimum value for $d$ according to the number $K$ of P2PSIP domains and the number $N$ of peers. One scenario where these parameters are given could be VoIP, $K$ is the number of groups that must be attached in the interconnection overlay in order to be reachable between them and $N$ is the overall number of peers.

In order to understand the importance of the number of dimensions $d$ on a Hierarchical CAN Overlay

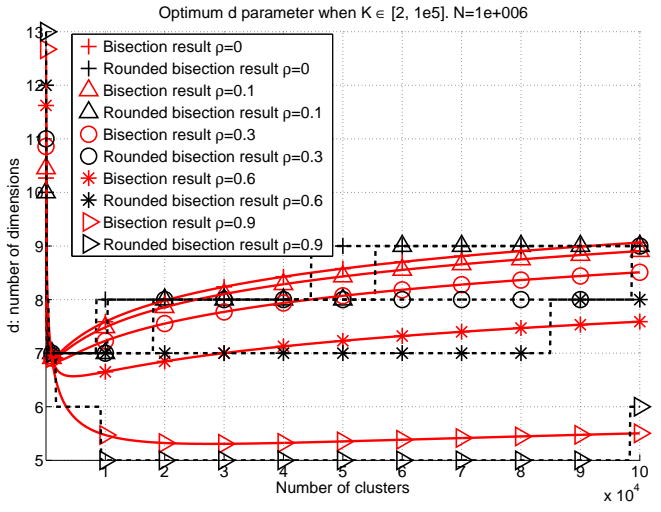

Figure 5. Optimum value of $\mathrm{d}$ depending on $\mathrm{K}$

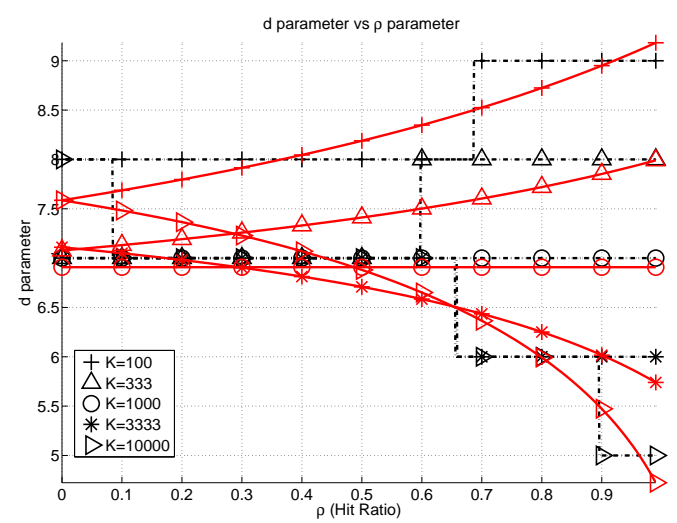

Figure 6. Dependency of $d$ parameter with $\rho_{i i}$

Network, Eq.16 has been solved using the numerical method of bisection. The value of $N=10^{6}$ has been used for this analysis and a large range of $K$ values has been explored. The results are shown in Fig.5, the solid line is the result of the bisection method and the dashed line is the nearest integer to the bisection results for each value of $K$. We can observe how the value of $d$ changes with $K$. It is interesting to note that the variation of $d$ is not a monotonic function with $K$ and it has a minimum value depending on the intra-domain hit ratio $\rho_{i i}$.

Fig.6 presents the dependency of $d$ with the intradomain hit probability. In solid lines is represented the result of the bisection method applied to different values of $\rho_{i i}$ for $N=10^{6}$ and fixed values of $K$. Dash-dot lines are used for the nearest integer values to the bisection results. The legend of the figure has the correspondence between the markers and the different values of $K$. If $K<\sqrt{N}$, the dependency of $d$ with respect to $\rho_{i i}$ is a monotonous increasing function. Whereas, if $K>\sqrt{N}$, the dependency of 


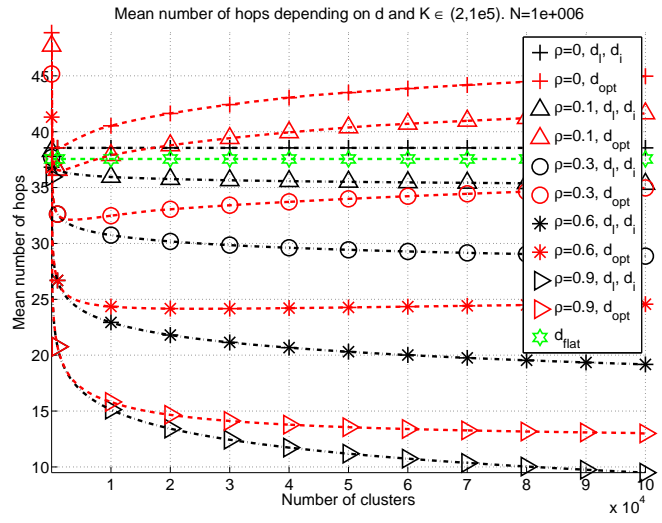

Figure 7. Routing Performance (Hops)

$d$ with respect to $\rho_{i i}$ is a monotonous decreasing function. Furthermore, for $K=\sqrt{N}$ the value of $d$ with respect to $\rho_{i i}$ is a constant. This is the point where the monotonous increasing dependency changes to monotonous decreasing.

When $\rho_{i i} \in(0,0.65)$, the value of $d \in[7,8]$ independently of $K$. This implies a wide range of work for $d$ if $\rho_{i i} \in(0,0.65)$. However, for higher values of $\rho_{i i}, d \in[5,9]$. Thus, more attention must be paid to $\rho_{i i}$ since the $d$ parameter has a smaller range of work.

Furthermore, in order to see the performance of a hierarchical CAN overlay, the Routing Performance complexity for an optimum flat CAN overlay is plotted in Fig.7 with a dashed line with stars $\left(d_{\text {flat }}=14\right)$. The Routing Performance for different values of the intradomain hit ratio is in dashdot lines when $d_{l}$ and $d_{i}$ are configured independently. On the other hand, the Routing Performance when an unique $d$ parameter is used is depicted with dashed lines. The values of $d$ were obtained from Fig.5. The key shows the different values of $\rho_{i i}$ that were used. Obviously, the Routing Performance is improved if the intra-domain hit ratio increases. A better Routing Performance than the flat counterpart can be obtained with small values of $\rho_{i i}$. Additionally, we obtain a smaller maintenance cost if we use the optimal values of $d$ from Fig.5. We can also observe how the difference between using an unique value of $d$ or a value of $d$ for each level decreases if the intra-domain hit ratio increases.

In addition we obtain a reduction of the state maintenance. This state is reduced from $d_{\text {flat }}$ overlay routing entries [14] to the values shown in Fig.5. The optimal value of $d$ for the Hierarchical CAN Overlay Network depends on the number of domains, the number of peers and the intra-domain hit ratio, but usually, for most of the cases, we get a reduction of the $50 \%$ in the maintenance state.

\section{Related Work}

Studies related with hierarchical overlay networks have been done to improve the performance of flat overlay networks. When a hierarchical architecture is considered, the different trade-offs that arise with these types of architecture have to be taken into account. Furthermore, these architectures have benefits [8], [9] in comparison with the flat ones.

One approach is to delegate all the work to superpeers [5], [20], which maintain the overlay network and perform all the necessary actions, peers only have to register their information to their super-peers. In our proposal, super-peers only have to store the information needed of the routing table for its P2PSIP domain, which is lighter $\left(\log M_{i}\right)$ and desirable for a more scalable solution.

Other studies focus on decrementing the delay in peer-to-peer overlay transactions. In [19], a low delay hierarchical overlay network based on Chord is proposed. The drawback is the high state maintenance needed (memory, CPU and bandwidth) because all the peers in the overlay are attached to all the levels in a $n$-level hierarchy. A less aggressive design with the same objective is presented in [4] where a hierarchical structure is built with the constraint of limiting the maintenance cost to the flat counterpart. The proposal in this paper only overloads super-peers, which is desirable because peers can be more lightweight. This feature is attractive for handheld devices that are candidates to use Skype-like applications in the future.

In relation with Hierarchical P2PSIP architectures, there is some work presented in [17]. However it does not provide a very detailed analysis on the Routing Performance and how to deal with non-fixed lengths in the Prefix and Suffix IDs needed for interoperability.

\section{Conclusions}

In this paper a Hierarchical DHT-based Overlay Architecture has been defined using the ongoing work in the IETF P2PSIP WG. In our proposal, there are different P2PSIP domains where an overlay is maintained using the P2PSIP protocol. Each domain has a super-peer that allows the connectivity with the rest of the domains through the Interconnection Overlay. The routing in each domain is based on a Suffix ID, whereas the routing in the Interconnection Overlay is based on the Prefix ID. If the Prefix ID and the Suffix ID are defined respectively as Prefix ID=hash (example.com) 
and Suffix ID=hash (user@example.com), a global Skype-like service can be provided between the different P2PSIP domains.

We provide a discussion on the Routing Performance depending on the intra-domain hit probability $\left(\rho_{i i}\right)$, which is the probability of doing calls inside the own domain. A reduction in the Routing Performance with respect to the flat counterpart is achieved since the hierarchical architecture takes advantage of the intradomain hit probability, which is not possible in a flat overlay network.

In the case of a Hierarchical CAN Overlay, the optimal value of $d$ for a given number of domains and values of $\rho_{i i}$ is obtained. Furthermore, we observe how the intra-domain hit ratio reduces the Routing Performance on a hierarchical CAN overlay network and how the optimal values of $d$ can be used in a wide range of work. Additionally, the complex relation between the optimal value for the number of dimensions $d$ and the value of the intra-domain hit probability and the number of groups $K$ is explained. We conclude that most of the cases, the maintenance costs is also reduced up to a $50 \%$ in comparison with the flat one.

In order to continue this work, simulations using the PeerFactSim.Kom [3] simulator are being developed.

\section{Acknowledgement}

This work has been supported by the European Union under the IST Content NoE (FP6-2006-IST038423), by the Regional Government of Madrid under the BioGridNet project (CAM, S-0505/TIC-0101) and by the Ministry of Science and Innovation under the CONPARTE project (MEC, TEC2007-67966-C0303/TCM).

\section{References}

[1] B. Beverly Yang and H. Garcia-Molina. Designing a super-peer network. In Data Engineering, 2003. Proceedings. 19th International Conference on, pages 49-60, 2003.

[2] D. Bryan, P. Matthews, E. Shim, and D. Willis. Concepts and terminology for peer to peer sip, July 2008. Internet Draft draft-ietf-p2psip-concepts-02.txt.

[3] V. Darlagiannis, A. Mauthe, N. Liebau, and R. Steinmetz. An Adaptable, Role-based Simulator for P2P Networks. Proceedings of the International Conference on Modeling, Simulation and Visualization Methods, pages 52-59, 2004.

[4] P. Ganesan, K. Gummadi, and H. Garcia-Molina. Canon in g major: designing dhts with hierarchical structure. In Distributed Computing Systems, 2004. Proceedings. 24th International Conference on, pages 263-272, 2004.
[5] L. Garces-Erice, E. W. Biersack, K. W. Ross, P. A. Felber, and G. Urvoy-Keller. Hierarchical p2p systems. In Proceedings of ACM/IFIP International Conference on Parallel and Distributed Computing (Euro-Par), 2003.

[6] S. Guha, N. Daswani, and R. Jain. An experimental study of the skype peer-to-peer voip system. In In IPTPS 2006.

[7] C. Jennings, B. Lowekamp, E. Rescorla, S. Baset, and H. Schulzrinne. Resource location and discovery (reload), July 2008. Internet Draft draft-ietf-p2psipreload-00.txt.

[8] M. Kwon and S. Fahmy. Toward Cooperative Interoverlay Networking. Proceedings of IEEE ICNP, 2003.

[9] M. Kwon and S. Fahmy. Synergy: an overlay internetworking architecture. In Computer Communications and Networks, 2005. ICCCN 2005. Proceedings. 14th International Conference on, pages 401-406, 2005.

[10] I. Martinez-Yelmo, R. Cuevas, C. Guerrero, and A. Mauthe. Routing performance in hierarchical dhtbased overlay networks. In In Proceedings on 16th Euromicro International Conference on Parallel, Distributed and network-based Processing, Feb. 2008.

[11] P. Maymounkov and D. Mazieres. IPTPS 2002 Cambridge, MA, USA, March 7-8, 2002. Revised Papers, volume 2429/2002 of Lecture Notes in Computer Science, chapter Kademlia: A peer-to-peer information system based on the XOR metric, pages 53-65. Springer, 2002.

[12] S.-H. Min, J. Holliday, and D.-S. Cho. Optimal superpeer selection for large-scale p2p system. In Hybrid Information Technology. ICHIT'06. Vol 2. International Conference on, volume 2, pages 588-593, 2006.

[13] A. T. Mizrak, Y. Cheng, V. Kumar, and S. Savage. Structured superpeers: leveraging heterogeneity to provide constant-time lookup. In Internet Applications. WIAPP. Proceedings., pages 104-111, 2003.

[14] S. Ratnasamy, P. Francis, M. Handley, R. Karp, and S. Schenker. A scalable content-addressable network. In SIGCOMM '01, pages 161-172, New York, NY, USA, 2001. ACM Press.

[15] J. Rosenberg. Interactive connectivity establishment (ice): A protocol for network address translator (nat) traversal for offer/answer protocols, October 2007. Internet Draft draft-ietf-mmusic-ice-19.txt.

[16] D. Rossi, M. Melia, and M. Meo. A detailed measurment of skype network traffic. In In IPTPS 2008, 2008.

[17] J. Shi, Y. Wang, L. Gu, L. Li, W. Lin, Y. Li, Y. Ji, and P. Zhang. A hierarchical peer-to-peer sip system for heterogeneous overlays interworking. Global Telecommunications Conference, 2007. GLOBECOM 'O7. IEEE, pages 93-97, Nov. 2007.

[18] H. Simon. The architecture of complexity. In M. Press, editor, The Sciences of the Artificial, pages 192-229. 1981.

[19] Z. Xu, R. Min, and Y. Hu. Hieras: a dht based hierarchical p2p routing algorithm. In Parallel Processing, 2003. Proceedings. International Conference on, 2003.

[20] S. Zoels, Z. Despotovic, and W. Kellerer. Cost-based analysis of hierarchical dht design. In Peer-to-Peer Computing. P2P 2006. Sixth IEEE International Conference on, pages 233-239, 2006. 